\title{
Decreased expression of ferroportin in prostate cancer
}

\author{
DONG XUE ${ }^{1}$, CUI-XING ZHOU ${ }^{1}, \mathrm{YUN}^{-B O} \mathrm{SHI}^{2}, \mathrm{HAO} \mathrm{LU}^{1}$ and XIAO-ZHOU HE \\ ${ }^{1}$ Department of Urology, Third Affiliated Hospital, Suzhou University, Changzhou, Jiangsu 213003; \\ ${ }^{2}$ Foreign Languages School, Changzhou Institute of Technology, Changzhou, Jiangsu 213002, P.R. China
}

Received September 11, 2014; Accepted May 7, 2015

DOI: 10.3892/ol.2015.3363

\begin{abstract}
The present study examined the expression levels of ferroportin, a transmembrane protein that transports iron from the inside of a cell to the outside, in the prostate cancer PC3, DU145 and LNCAP cell lines, in the normal prostate RWPE2 cell line, and in tissue samples from different differentiation stages of prostatic carcinoma and prostatic hyperplasia. The study also investigated the role of ferroportin protein expression in the diagnosis and prognosis of prostate cancer. Reverse transcription-quantitative polymerase chain reaction and western blot analysis were employed to measure the mRNA and protein expression levels of ferroportin in the PC3, DU145, LNCAP and RWPE2 cells. Immunohistochemistry was used to determine ferroportin protein expression in the prostate cancer and prostatic hyperplasia tissues. Compared with the normal prostate RWPE2 cells, ferroportin protein expression was significantly lower in the prostate cancer PC3, DU145 and LNCAP cells $(\mathrm{P}<0.05)$. Compared with the prostatic hyperplasia tissues, ferroportin protein expression was significantly reduced in the prostate cancer tissues $(\mathrm{P}<0.05)$. Overall, the expression levels of ferroportin in the prostate cancer tissues were lower than those in the normal prostate tissues, which may provide valuable clinical information for the diagnosis and prediction of disease progression in prostate cancer, and may indicate a potential therapeutic target for treating prostate cancer by regulating iron metabolism.
\end{abstract}

\section{Introduction}

Prostate cancer is the most common malignancy in males in western countries, and the second most common cause of cancer-related mortality (1). The clinical symptoms of early prostate carcinoma are unspecific, and the disease is therefore often diagnosed at a late stage. With the extensive use of serum

Correspondence to: Professor Xiao-Zhou He, Department of Urology, Third Affiliated Hospital, Suzhou University, 185 Juqian Street, Changzhou, Jiangsu 213003, P.R. China E-mail: hexz911@163.com

Key words: prostate cancer, benign prostatic hyperplasia, hepcidin, ferroportin prostate-specific antigen detection and biopsy of the prostate, the early diagnosis rate of prostate cancer has improved $(2,3)$.

A previous study found that iron metabolism plays a significant role in cancer cell growth, angiogenesis and metastasis (4). Hepcidin, predominantly synthesized in the liver, is the principal regulator of systemic iron homeostasis, and acts by inhibiting intestinal iron absorption, iron recycling by macrophages, and iron mobilization from hepatic stores (5). It has been reported that hepcidin is closely associated with infection, tumor and chronic inflammation (6). Ferroportin protein is an important regulator of body iron metabolism, and is a membrane transport protein that transfers intracellular iron to the extracellular environment. Reduced expression levels of ferroportin on the cell surface lead to an increase in intracellular free iron, making the tumor cells more aggressive. Changes in ferroportin protein expression caused by abnormal iron metabolism often induce reactions in tumor invasion and metastasis (7). Ferroportin has been reported to be significantly correlated with prognosis in breast cancer (8). However, thus far, the role of ferroportin protein expression in prostate cancer remains elusive.

The present study analyzed the expression levels of ferroportin protein in different differentiation stages of prostate cancer and prostate hyperplasia, as well as the differences in prostate cancer and normal prostate cells.

\section{Materials and methods}

Subjects. The subjects of the present study were selected from 60 patients with prostate cancer and 30 patients with benign prostatic hyperplasia $(\mathrm{BPH})$ who visited the Third Affiliated Hospital (Suzhou University, Changzhou, Jiangsu, China) between January 2008 and December 2012. The study was approved by the Ethics Committee/Institutional Review Board of the hospital, and was performed in accordance with the Declaration of Helsinki. Written informed consent was obtained from all patients. The age range of the patients was 55-75 years, with a mean of 67 years. Prostate cancer was pathologically diagnosed in the 60 cancer patients. According to the Gleason score (9), 20 cases presented with scores of $<7$, 15 cases with scores of 7 and 25 cases with scores of $>7$. For the remaining 30 subjects, BPH was diagnosed by a transurethral resection of the prostate pathology.

Ferroportin protein was measured by immunohistochemistry. Surgical specimens were fixed in formalin and embedded in paraffin blocks. Sections (4- $\mu \mathrm{m}$ thick) were incubated for $1 \mathrm{~h}$ 
Table I. Primers for ferroportin and GAPDH.

\begin{tabular}{lllc}
\hline Genes & \multicolumn{1}{c}{ 5'-Primers } & \multicolumn{1}{c}{ 3'-Primers } & Products, bp \\
\hline Ferroportin & AGCCTGCCACCACCAACCCGTAGA & TGGCTCCCAGGACCAGAAC & 225 \\
GAPDH & CAAGGTCATCCATCCATGACAACTTTG & GTCCACCACCCTGTTGCTGTAG & 496 \\
\hline
\end{tabular}
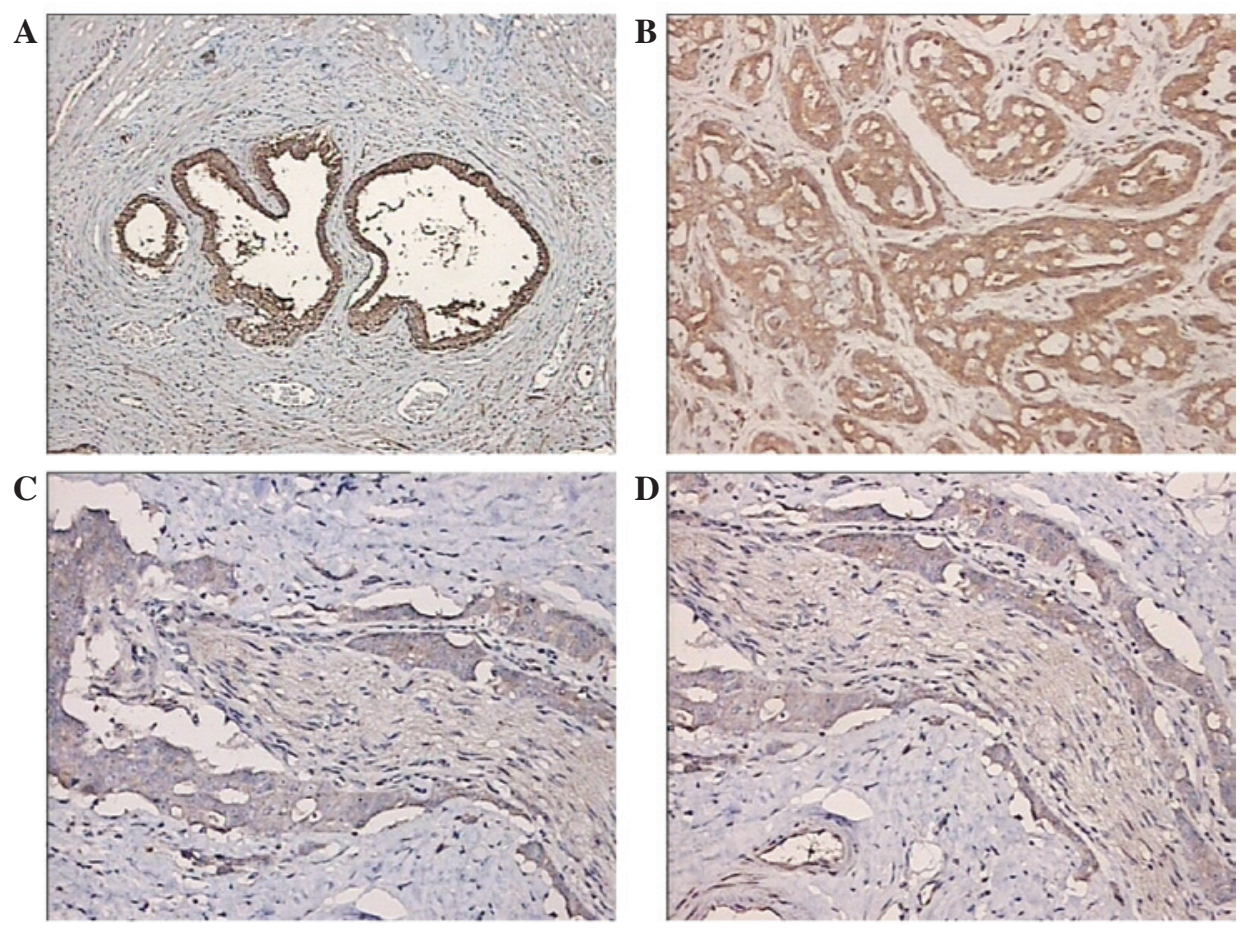

Figure 1. Expression of ferroportin protein in tissue samples, as determined by immunohistochemistry. (A) Benign prostatic hyperplasia tissue samples; (B) highly-differentiated prostate cancer (Gleason score, <7); (C) moderately-differentiated prostate cancer (Gleason score, 7); and (D) poorly-differentiated prostate cancer (Gleason score, $>7$ ) (x200 magnification).

at $60^{\circ} \mathrm{C}$, heated in an oven at $37^{\circ} \mathrm{C}$ for $15 \mathrm{~min}$, de-paraffinized and rehydrated using serial xylene and ethanol (Sigma-Aldrich, St. Louis, MO, USA) incubations, then transferred to sodium citrate buffer (pH 6.0; Sigma-Aldrich) for 15 min. Following antigen retrieval, the sections were incubated in $3 \%$ peroxide bicarbonate solution at room temperature for $10 \mathrm{~min}$ to block endogenous peroxidase activity. Ferroportin protein expression was detected using a horseradish peroxidase (HRP) complex with a rabbit monoclonal anti-ferroportin primary antibody (1:50; cat. no. ab85370; Abcam, Cambridge, MA, USA), visualized using 3,3'-diaminobenzidine staining and observed by microscope (Olympus BHS; Olympus Corporation, Tokyo, Japan) (Fig. 1).

Ferroportin gene expression measured by reverse transcription-quantitative polymerase chain reaction (RT-qPCR). The prostate cancer PC3, DU145 and LNCAP cell lines, and the normal prostate RWPE2 cell line were obtained from Shanghai Institutes for Biological Sciences, Shanghai, China). The cells were cultured in RMPI 1640 medium supplemented with $10 \%$ fetal bovine serum, $100 \mathrm{U} / \mathrm{ml}$ penicillin and $100 \mathrm{U} / \mathrm{ml}$ streptomycin (Sigma-Aldrich) in an atmopshere of $5 \% \mathrm{CO}_{2}$ at $37^{\circ} \mathrm{C}$. RNA was extracted with TRIzol (Invitrogen Life Technologies,
Carlsbad, CA, USA). RT was performed according to the manufacturer's instructions by adding oligo(dT), 5X Reaction Buffer, RNase Inhibitor, $10 \mathrm{mM}$ dNTP mix and M-MuLV reverse transcriptase sequentially (PCR supermix; catalog no. 10572-014; Invitrogen). The Luminaris Color HiGreen qPCR Master Mix PCR kit was purchased from Thermo Fisher Scientific, Inc., (Waltham, MA, USA) and the RT-PCR reactions were performed at $94^{\circ} \mathrm{C}$ for $5 \mathrm{~min}, 94^{\circ} \mathrm{C}$ for $20 \mathrm{sec}$, $55^{\circ} \mathrm{C}$ for $20 \mathrm{sec}$ and $72^{\circ} \mathrm{C} 15 \mathrm{sec}$ for 30 cycles, followed by $72^{\circ} \mathrm{C}$ for $5 \mathrm{~min}$. The sequences of the primers are presented in Table I. PCR products were transferred to $2 \%$ agarose gel and the band intensity was quantified using the Kodak Gel Logic 200 imaging system (Kodak, Rochester, NY, USA).

Ferroportin protein expression measured by western blotting. Cells grown in a $25-\mathrm{cm}^{2}$ flask were rinsed with PBS prior to harvesting. Total proteins were extracted with a cell lysis buffer (Pierce Biotechnology, Inc., Rockford, IL, USA) containing phenylmethylsulfonyl fluoride and incubated on ice for $10 \mathrm{~min}$. The lysate was separated by centrifugation at $100,000 \mathrm{x}$ g for $30 \mathrm{~min}$. The amount of protein was measured by bicinchoninic acid assay (Pierce Biotechnology, Inc.). For $12 \%$ SDS-PAGE, a total of $20 \mu \mathrm{g}$ of protein was loaded 


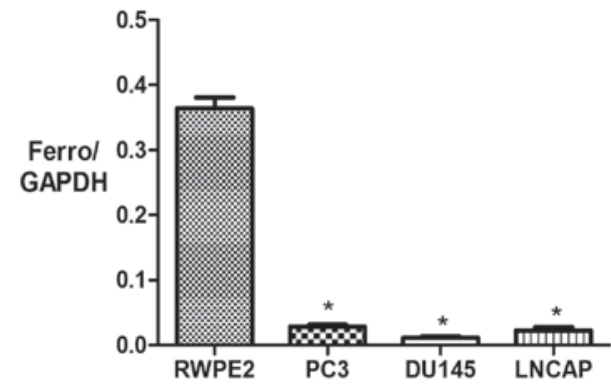

Figure 2. mRNA expression levels of ferroportin (Ferro) in the prostate cancer cell lines PC3, DU145 and LNCAP, and the normal prostate cell line RWPE2, as determined by reverse transcription-quantitative polymerase chain reaction. ${ }^{*} \mathrm{P}<0.05$ vs. RWPE2.

A

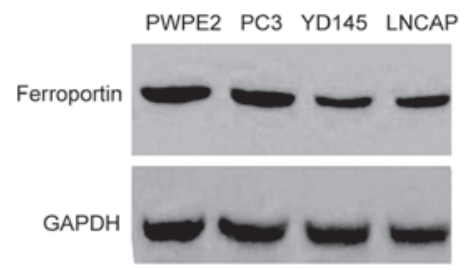

B

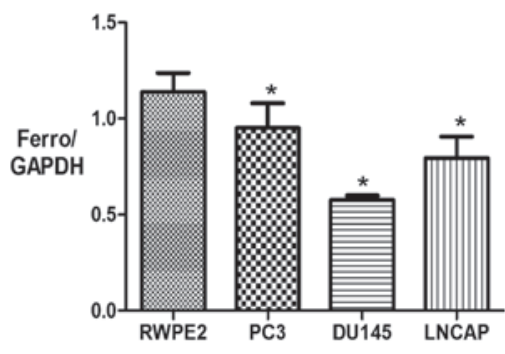

Figure 3. Expression of ferroportin (Ferro) in the prostate cancer cel lines PC3, DU145 and LNCAP, and the normal prostate cell line RWPE2. (A) Western blot analysis and (B) quantification of western blotting bands. ${ }^{*} \mathrm{P}<0.05$ vs. RWPE2.

per well. Polyvinylidene difluoride (PVDF) membranes (Roche Diagnostics, Indianapolis, IN, USA) were used for the transfer process. For western blotting, PVDF membranes were incubated in 5\% skimmed milk blocking buffer for $1 \mathrm{~h}$, followed by washing 3 times with Tris-buffered saline plus $0.1 \%$ Tween 20 . Ferroportin was detected using a rabbit monoclonal ferroportin-specific antibody (1:500; cat. no. ab85370) and horseradish peroxidase-conjugated rabbit anti-mouse IgG secondary antibodies (1:2,000; cat. no. ab6728; Abcam). Mouse monoclonal GAPDH (1:2,000; cat. no. ab130099; Abcam) was used as a loading control. The protein bands were developed using the Electrochemiluminescence Plus kit (GE Healthcare, Bio-Sciences, Pittsburgh, PA, USA), and band intensity was quantified using ImageJ (http://imagej. nih.gov/ij/).

Statistical analysis. Statistical analyses were performed using SPSS version 13.0 (SPSS, Inc., Chicago, IL, USA). Data were analyzed by homogeneity of variance tests and are expressed as the mean \pm standard deviation. Differences were analyzed by t-test. Pearson correlation was used to analyze the association between all studied parameters. For heterogeneity of variance, non-parametric tests were used, whereas the Mann-Whitney U test was used for pairwise comparisons. $\mathrm{P}<0.05$ was used to indicate a statistically significant difference.

\section{Results}

Expression levels of ferroportin in prostate cancer. The expression levels of ferroportin were examined by immunohistochemical analysis in the prostate cancer and prostatic hyperplasia tissue samples (Fig. 1). Reduced ferroportin expression levels were found in the prostate cancer tissue samples. With the decline in prostate cancer cell differentiation and increased degree of malignancy, ferroportin expression was reduced and exhibited less immunostaining in the cytoplasm. Moreover, the prostatic hyperplasia tissue samples exhibited increased ferroportin immunostaining suggesting enhanced expression levels.

$m R N A$ expression of ferroportin in cancer cell lines. RT-qPCR was used to measure mRNA expression levels of ferroportin in the prostate cancer PC3, DU145 and LNCAP cell lines compared with the normal prostate RWPE2 cell line. GAPDH was used as an internal standard to normalize the data. The mRNA expression levels of ferroportin were significantly lower in the prostate cancer PC3, DU145 and LNCAP cell lines $(0.028 \pm 0.004,0.011 \pm 0.002$ and $0.022 \pm 0.008$, respectively) compared with the normal prostate RWPE2 cell line $(0.36 \pm 0.03)(\mathrm{P}<0.05$; Fig. 2).

Expression offerroportin in prostate cancer cell lines. Western blotting was employed to measure the protein expression levels of ferroportin in the prostate cancer PC3, DU145 and LNCAP cell lines and the normal prostate RWPE2 cell line. Consistent with the findings of the mRNA expression levels, the protein expression levels of ferroportin were significantly lower in the prostate cancer PC3, DU145 and LNCAP cell lines $(0.95 \pm 0.22$, $0.57 \pm 0.04$ and $0.79 \pm 0.19$, respectively) compared with the normal prostate RWPE2 cell line $(1.13 \pm 0.17)(\mathrm{P}<0.05)$ (Fig. 3).

\section{Discussion}

Numerous cancer cells exhibit an increased demand for iron (10). Consequently, proteins involved in iron regulation are often deregulated in cancers and play a significant role during the tumor development process. Ferroportin is a transmembrane protein that transports iron from the inside of cells to the outside, and is the only known mammalian iron exporter to date. High levels of ferroportin protein have been detected in intestinal epithelial cells, placenta cells, liver cells and macrophages regulating iron export by the hepcidin-ferroportin axis (11). Previous studies have demonstrated that the expression levels of ferroportin and hepcidin affect the growth and progression of breast cancer cells, and that the stability of ferroportin is regulated by hepcidin (12). Compared with normal breast epithelial cells, the expression level of hepcidin is increased in breast cancer cells, whereas ferroportin exhibits reduced expression, which is associated with reduced iron export leading to enhanced iron availability to the tumor cells. Moreover, another previous study also found that decreased levels of ferroportin 
gene expression are associated with a significant reduction in metastasis-free and disease-specific survival that is independent of other breast cancer risk factors (12). It has been reported that the ferroportin protein is a strong and independent predictor of prognosis in breast cancer, and a novel potential target for molecular therapy (13). However, only two studies have reported that hepcidin is closely associated with anemia in patients with advanced prostate cancer (14). In the present study, the expression levels of ferroportin were measured in prostate tissues and prostate cancer cell lines; the results suggested that the decreased hepcidin-ferroportin axis may involved, as hepcidin predominantly mediates the degradation of ferroportin (15).

According to the Gleason score, prostate cancer tissue samples were divided into highly-differentiated, moderately-differentiated and poorly-differentiated prostatic hyperplasia groups and a normal group. The expression levels of ferroportin protein were measured by immunohistochemistry in each group, and reduced ferroportin protein expression was found in the prostate cancer tissues. With decreased prostate cancer cell differentiation and an increased degree of malignancy, the expression levels of ferroportin protein were decreased, together with less ferroportin immunostaining in the cytoplasm. By contrast, the expression level of ferroportin was increased in BPH. The different expression levels of ferroportin protein in the prostate cancer and BPH tissues at different differentiation statuses suggested that the ferroportin protein is closely associated with the prostate cancer cell development process.

In order to further investigate whether ferroportin protein regulates the development process of prostate cancer, the mRNA and protein expression levels of ferroportin were compared in the prostate cancer PC3, DU145 and LNCAP cell lines, and the normal prostate RWPE2 cell line. RT-qPCR analysis suggested that the mRNA levels of ferroportin were lower compared with the control group, whereas there was no marked difference between the prostate cancer cell lines. This observation was confirmed by western blotting.

To the best of our knowledge, the present study is the first to reveal and compare the expression levels of ferroportin protein in prostate cancer cells and normal prostate cells. We believe that ferroportin protein plays a significant role in the development of prostate cancer. It is possible that decreased expression levels of ferroportin in tumor cells inhibits intracellular iron export, causing intracellular iron overload and activating reactive oxygen species, consequently inducing DNA damage and impairing proteins and lipids, leading to tumorigenesis. Increased intracellular iron further promotes the growth of tumor cells (16). Collectively, data from the present study and other studies suggests that ferroportin protein could be a potential indicator for the diagnosis and prognosis of patients with prostate cancer. Moreover, ferroportin protein has clinical significance in prostate cancer as a potential target for molecular-targeted therapy by regulating cellular iron metabolism, and inhibiting the growth and progression of prostate cancer.

\section{References}

1. Ye L, Kynaston HG and Jiang WG: Bone metastasis in prostate cancer: Molecular and cellular mechanisms. Int J Mol Med 20: 103-111, 2007.

2. Aihara M, Lebovita RM, Wheeler TM, Kinner BM, Ohori M, and Scardino PT: Prostate specific antigen and gleason grade: An immunohistochemical study of prostate cancer. J Urol 151: 1558-1564, 1994.

3. Melchior SW and Brawer MK: Role of transrectal ultrasound and prostate biopsy. J Clin Ultrasound 24: 463-471, 1996.

4. Torti SV and Torti FM: Ironing out cancer. Cancer Res 71: 1511-1514, 2011.

5. Nemeth E and Ganz T: Regulation of iron metabolism by hepcidin. Annu Rev Nutr 26: 323-342, 2006.

6. Nemeth E, Rivera S, Gabayan V, Keller C, Taudorf S, Pedersen BK and Ganz T: IL-6 mediates hypoferremia of inflammation by inducing the synthesis of iron regulatory hormone hepcidin. J Clin Invest 113: 1271-1276, 2004.

7. Pogribny IP: Ferroportin and hepcidin: a new hope in diagnosis, prognosis and therapy for breast cancer. Breast Cancer Res 12: $314,2010$.

8. Pinnix ZK, Miller LD, Wang W, et al: Ferroportin and iron regulation in breast cancer progression and prognosis. Sci Transl Med 2: 43-56, 2010.

9. Gleason DF: Histologic grading of prostatic carcinoma. In: Pathology of the prostate (Contemporary Issues in Surgical Pathology). Bostwick MD and David G (eds). Churchill Livingstone, New York, pp83-87, 1990.

10. Cairo G, Bernuzzi F and Recalcati S: A precious metal: Iron, an essential nutrient for all cells. Genes Nutr 1: 25-39, 2006.

11. Merlot AM, Kalinowski DS and Richardson DR: Novel chelators for cancer treatment: Where are we now? Antioxid Redox Signal 18: 973-1006, 2013.

12. Pinnix ZK, Miller LD, Wang W, D'Agostino R Jr, Kute T, Willingham MC, Hatcher H, Tesfay L, Sui G, Di X, et al: Ferroportin and iron regulation in breast cancer progression and prognosis. Sci Transl Med 2: 43-56, 2010.

13. Song S, Christova T, Perusini S, Alizadeh S, Bao RY, Miller BW, Hurren R, Jitkova Y, Gronda M, Isaac M, et al: Wnt inhibitor screen reveals iron dependence of $\beta$-catenin signaling in cancers. Cancer Res 71: 7628-6737, 2011.

14. Tanno T, Rabel A, Alleyne M, Lee YT, Dahut WL, Gulley JL and Miller JL: Hepcidin, anaemia and prostate cancer. BJU Int 107: 678-9, 2011.

15. De Domenico I, Ward DM, Langelier C, Vaughn MB, Nemeth E, Sundquist WI, Ganz T, Musci G and Kaplan J: The molecular mechanism of hepcidin-mediated ferroportin down-regulation. Mol Biol Cell 18: 2569-2578, 2007.

16. Ellervik C, Tybjaerg-Hansen A and Nordestgaard BG: Risk of cancer by transferrin saturation levels and haemochromatosis genotype: Population-based study and meta-analysis, J Intern Med 271: 51-63, 2012. 